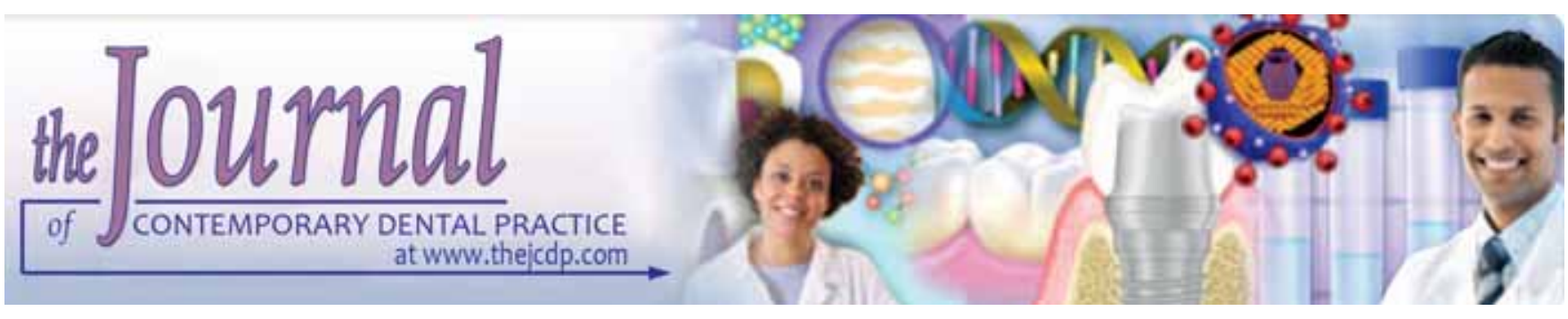

\title{
Modified Gap Arthroplasty and Myrhaug's Incision as a Treatment Option in Management of Temporomandibular Joint Ankylosis: A Study of 10 Cases
}

Arvind Agarwal, Brijesh Ruparelia, Ajay Kubawat, Sandeep Patel, Shalini Gupta

\begin{abstract}
Purpose of the study: The purpose of this study was to evaluate effectiveness of modified gap arthroplasty procedure and modified Myrhaug's preauricular incision for treating bony temporomandibular joint (TMJ) ankylosis.
\end{abstract}

\begin{abstract}
Materials and methods: A total of 10 cases were included in the study with Sawhney's type III and IV TMJ ankylosis. Study consisted of 6 males and 4 females. Three cases were treated for bilateral ankylosis and 7 were treated for unilateral ankylosis. All the patients were treated with modified gap arthroplasty and modified Myrhaug's incision was used. In this modification, osteotomy cuts were modified in such a way that coronoid process was also removed simultaneously along with the osteotomized ankylosed mass in one piece.
\end{abstract}

Results: All the patients were followed up for 5 years and none of them presented with recurrence. Two patients had transient paresis to zygomatic and temporal branch of facial nerve and none had permanent damage to the nerve. Seven patients had mouth opening more than $25 \mathrm{~mm}$ (p) when followed for 5 years. Anterior open bite was seen in 4 patients and deviation of the jaw on ipsilateral side was noted in 2 patients.

Conclusion: Modified gap arthroplasty gave successful results in the follow-up period and eliminated the need of separate coronoidectomy. Use of modified Myrhaug's preauricular approach, provided excellent visibility particularly of the elongated coronoid process, minimized bleeding, prevented damage to the vital anatomical structures surrounding the joint and gave acceptable cosmetic results.

Clinical significance: The modified incision has been found to be reducing intra- and postoperative morbidity as well as provides excellent accessibility. The modified osteotomy technique eliminated the need for separate coronoidectomy thus significantly reducing the operating time.

Keywords: Modified gap arthroplasty, Modified Myrhaug's preauricular incision, Sawhney's type III and IV ankylosis, Coronoidectomy.

How to cite this article: Agarwal A, Ruparelia B, Kubawat A, Patel S, Gupta S. Modified Gap Arthroplasty and Myrhaug's Incision as a Treatment Option in Management of Temporomandibular
Joint Ankylosis: A Study of 10 Cases. J Contemp Dent Pract 2011;12(4):295-300.

Source of support: Nil

Conflict of interest: None declared

\section{INTRODUCTION}

Ankylosis of the temporomandibular joint (TMJ) is an intracapsular union of the disk-condyle complex to the temporal articular surface that restricts mandibular movements, including the fibrous adhesions or bony fusion between condyle, disk, glenoid fossa and eminence. ${ }^{1}$

Ankylosis, developing in childhood or the early stages of development is most commonly due to trauma..$^{2-5}$ It is a serious and disabling condition that may cause problems in mastication, digestion, speech, appearance and hygiene. It can also causes disturbances of facial and mandibular growth, and acute compromise of the airway invariably resulting in physical and psychological disability. ${ }^{6,7}$ This factor makes the surgical intervention mandatory at the earliest to allow the patient to lead a physically and psychologically healthy life. Surgical techniques most commonly employed for this disease includes gap arthroplasty with or without costochondral grafting or placement of interpositional material to prevent reankylosis. ${ }^{2,4,8,9}$

The treatment of TMJ ankylosis poses a significant challenge because of technical difficulties and a high incidence of recurrence. A variety of techniques for its treatment have been described in the literature. However, no single method has produced uniformly successful results. ${ }^{2,10,11}$

The aim of this study was to evaluate effectiveness of modified gap arthroplasty procedure and modified Myrhaug’s preauricular incision for treating bony TMJ 
ankylosis. The coronoid process which is invariably elongated is removed simultaneously along with the osteotomized ankylosed mass in one piece, eliminating the need for separate coronoidectomy. ${ }^{10}$ Postoperatively vigorous mouth opening exercises were advised under strict supervision. All the cases were followed up for a period of 5 years by clinical and radiological evaluation.

\section{MATERIALS AND METHODS}

Ten cases of intraarticular bony ankylosis of TMJ were treated with modified gap arthroplasty procedure, were included in the study. Diagnosis in all cases was made on the basis of a detailed clinical history as well as radiological findings. Computed tomography (CT) scans were taken in all the cases to determine the extent of ankylosed masses requiring dissection (Fig. 1). The Sawhney criteria were taken as the basis of classification ${ }^{12}$ and type III and type IV were included in the study. Study consisted of 6 males and 4 females. Three cases were treated for bilateral ankylosis and 7 were treated for unilateral ankylosis. Written consent was taken from the patients after explaining the procedure in detail. Blind nasal intubation was successful in 5 patients. Tracheostomy was performed in 2 patients. In 3 patients, intubation was carried out with flexible fiberoptic laryngoscope to administer general anesthesia. The data were analyzed using the test to correlate significance.

\section{INCISION}

In all the cases, the joint was approached by modified Myrhaug's preauricular skin incision (Fig. 2). Here, Myrhaug's incision was modified by extending the anterior part of its horizontal extension more forwards and downwards to prevent nerve damage and better surgical exposure.

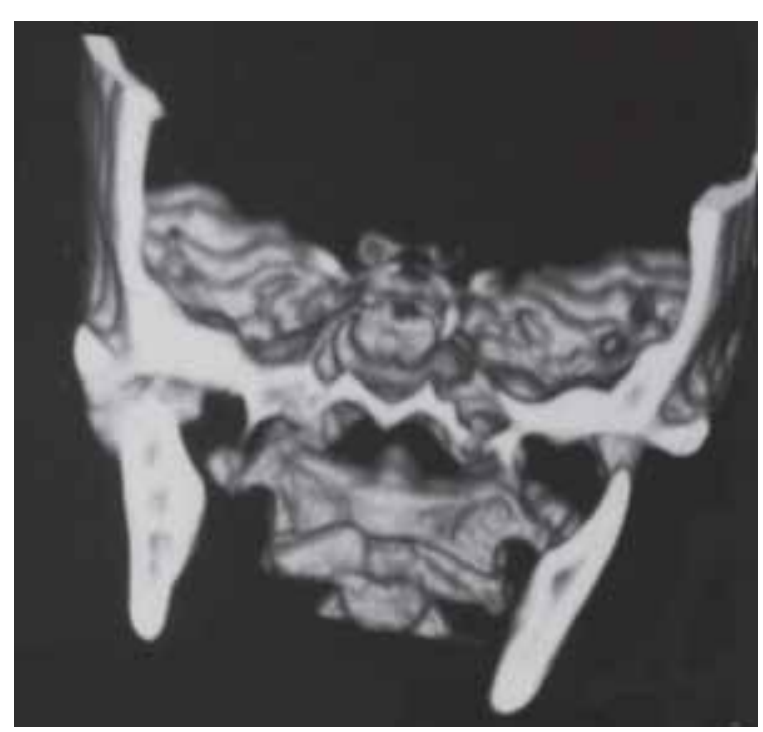

Fig. 1: Preoperative 3D CT scan showing ankylosed TMJ

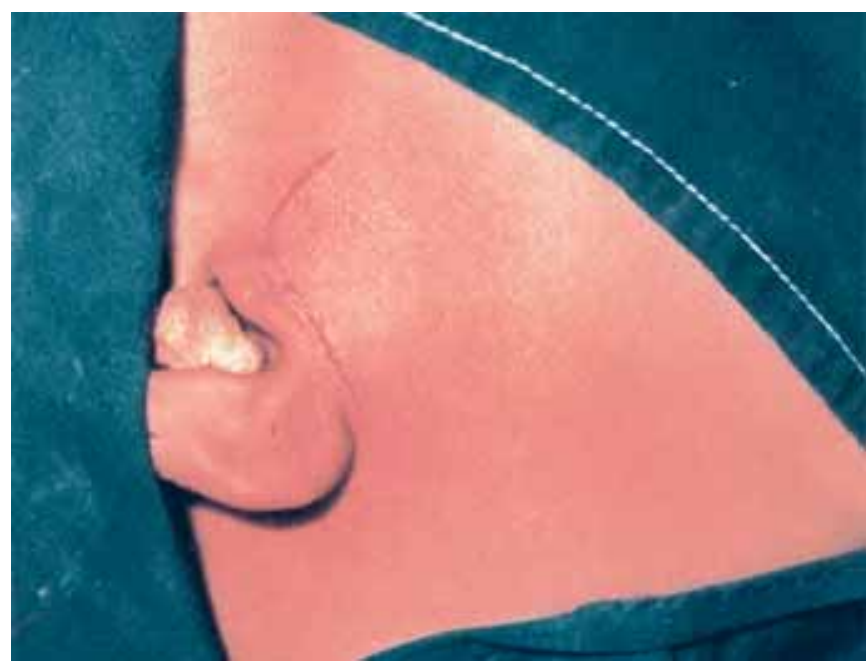

Fig. 2: Modified Myrhaug's preauricular incision

\section{OSTEOTOMY CUTS}

It is imperative to identify the bony landmarks like sigmoid notch, the posterior and the anterior border of ramus. First, the lower osteotomy cut was made below the sigmoid notch starting from the anterior to the posterior border. Approximately, two-third thickness of the bone was cut with the bur and the remaining one-third of the bone was split with an osteotome to prevent damage to the internal maxillary artery. The second osteotomy cut was made approximately at a distance of $1.5 \mathrm{~cm}$ above the lower cut and extended from the lower edge of sigmoid notch from anterior to the posterior border of ramus diverging posteriorly from the first cut. This modification of the standard gap arthroplasty procedure eliminated the need for separate coronoidectomy and allowed the ankylosed mass along with elongated coronoid process to be removed in one piece (Fig. 3). Mouth opening was checked using

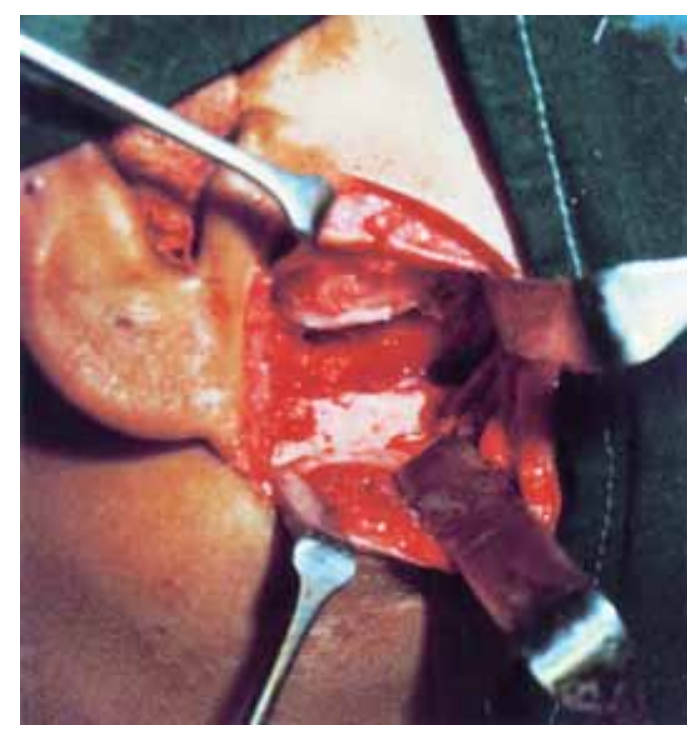

Fig. 3: Surgical exposure of the ankylosed region with osteotomized gap 
Ferguson's mouth gag. Free and unhindered movement of the mandible was ascertained before closure of wound in layers with 3-0 Vicryl sutures. Finally, skin sutures were taken with 3-0 Mersilk. In all 10 cases, vigorous postoperative physiotherapy was instituted for a period of 3 to 6 months.

\section{RESULTS}

In our study, 9 patients were above 10 years and in 7 duration of ankylosis was more than 6 years. Out of 10 patients, in 6 trauma was the etiological factor and other factors were infection in 3 and unknown etiology in 1 . Total joints treated were 13, in which 7 patients had unilateral and 3 had bilateral ankylosis. Preoperative mouth opening in 5 was less than $1 \mathrm{~mm}$ (Table1). Significance value?

All the patients were followed up for 5 years and none of them presented with recurrence. Two patients had paresis to zygomatic and temporal branch of facial nerve in the immediate postoperative period and none had permanent damage to the nerve and 7 patients had mouth opening more than $25 \mathrm{~mm}$ when followed for 5 years. Anterior open bite was seen in 4 patients and deviation of the jaw on ipsilateral side was noted in 2 patients (Table 2).

\section{DISCUSSION}

In this study, etiological factor for ankylosis was trauma in 6 out of 10 patients, whereas infection was the second most common cause in 3 patients. In the present series, 9 out of 10 patients were afflicted by this disease before the age of 10 years.

One of the interesting finding of this study was that all patients of this series sought treatment after an average period of 9 years from the onset of disease. Occurrence of TM joint ankylosis at an early age and the delayed treatment sought by the patient may be the principle factors responsible for severe facial deformity. This deformity becomes more pronounced in bilateral cases. In the present series, 3 cases presented with bilateral ankylosis resulting in bird face appearance, whereas rest 7 cases were of unilateral ankylosis with facial deformity and deviation of lower third of face to the affected side.

The principle surgical procedures currently used are gap arthroplasty, interpositional arthroplasty and condylectomy with costochondral grafting particularly in children., ${ }^{2,4,8,13}$ Gap arthroplasty alone gives rise to a gap between the articular cavity and the mandibular ramus and has the advantage of simplicity and short operating time. ${ }^{2}$ Vasconcelos et al ${ }^{14}$ reported 8 cases of ankylosis (type I to IV) treated by gap arthroplasty and found no recurrence in their series with a follow-up of at least 24 months. In our study, none of the patients were seen with recurrence after follow-up for 5 years.

Comparion of gap arthroplasty and interpositional gap arthroplasty on the temporomandibular joint ankylosis, the results showed that the range of mouth opening significantly increases after ankylosis surgery in both surgical approaches but improvement was less in interpositional gap arthroplasty. In regards to recurrence, results of interpositional gap arthroplasty were superior to gap arthroplasty. ${ }^{15}$

Interpositional arthroplasty with autogenous or alloplastic material at the osteotomy site is a mechanism for preventing recurrence. ${ }^{2,11}$ However, there are possible disadvantages, such as morbidity at the donor site and unpredictable resorption, muscle shrinkage, fibroses, fascia lacking bulk, cartilage tending to fibrose and calcify when autogenous material is used, and a risk of foreign body reaction and disintegration under functional loads when alloplastic material is used. ${ }^{2,3,6,16-19}$ Thus, at present, there is no ideal interpositional graft. In the current study however, interpositional graft was not used.

In the present series all the patients were diagnosed with the help of TM joint view X-rays, orthopantomograms and computed tomograms of both the affected and unaffected joints. All of these patients showed the classical radiological findings of ankylosis. A noticeable finding in 3 patients

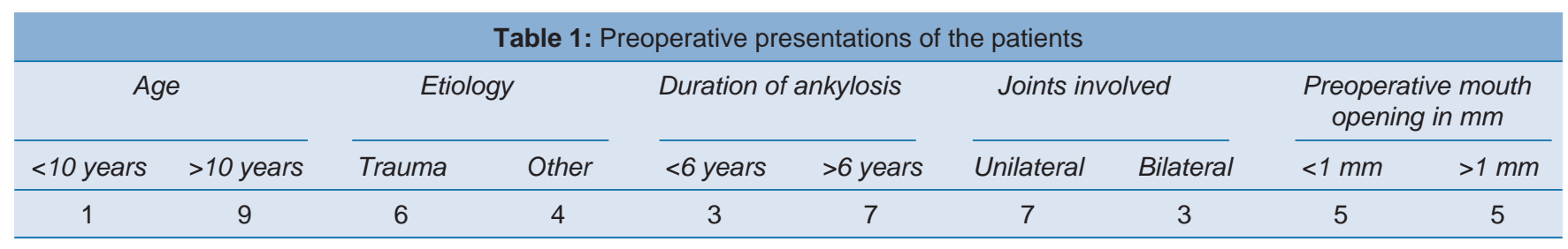

\begin{tabular}{|c|c|c|c|c|c|c|c|c|}
\hline \multicolumn{2}{|c|}{$\begin{array}{l}\text { Postoperative } \\
\text { mouth opening } \\
\text { in } \mathrm{mm}\end{array}$} & \multicolumn{2}{|c|}{ Anterior open bite } & \multirow{2}{*}{$\begin{array}{c}\text { Recurrence after } \\
5 \text { years }\end{array}$} & \multicolumn{2}{|c|}{$\begin{array}{c}\text { Paresis to zygomatic/ } \\
\text { temporal branch of } \\
\text { facial nerve }\end{array}$} & \multicolumn{2}{|c|}{$\begin{array}{l}\text { Deviation of jaw on } \\
\text { ipsilateral side }\end{array}$} \\
\hline$<25 \mathrm{~mm}$ & $>25 \mathrm{~mm}$ & Present & Absent & & Present & Absent & Present & Absent \\
\hline 3 & 7 & 4 & 6 & 0 & 2 & 8 & 2 & 8 \\
\hline
\end{tabular}


was of the complete obliteration of the sigmoid notch by the hypertrophied bony mass and even outline of the coronoid process could not be made out. Another finding was that of an unerupted tooth quite high up in the ramus in 2 of the cases due to the reduced ramus height. These findings had to be taken into consideration during surgery while planning for osteotomy cuts.

In one of the patient of this series, CT scan along with 3D scans was performed. It provided excellent information about the ankylosed mass in all the three planes viz coronal, sagittal and axial. It also provided information of the location of major anatomic structures like internal maxillary artery, parotid gland, internal carotid artery, external jugular vein, thus helping the surgeon to avoid them. ${ }^{20,21}$

Irrespective of the technique chosen by the surgeon, aggressive resection of the bony or fibrous ankylotic segment is crucial to avoid recurrence. ${ }^{2}$ In addition, a dissection of the muscles of the mandibular ramus and ipsilateral coronoidectomy must be carried out to prevent inadequate intraoperative interincisal opening, because the coronoid process may be elongated in long-standing cases. ${ }^{22}$ In our case series, duration of ankylosis in 7 of the cases was more than 6 years. So, resection of the ankylosed mass along with ipsilateral coronoid process was planned and this was carried out with a single osteotomy cut.

While making osteotomy cuts, care was taken that the cuts diverged posteriorly so as to prevent the posterior ends of the bony stumps from coming in contact, while the mouth is opened. Another modification done in this study was, if sigmoid notch or a part of it was present, lower cut was made as usual from anterior to the posterior border of the ramus just below the sigmoid notch, however the upper cut was made in the region of the condylar neck, starting from just above the lowest point of the sigmoid notch from the anterior to the posterior border of the neck of the condyle upwards. This modification eliminated the need of making a separate cut for coronoidectomy. Here, coronoid process and the cut bone could be removed in one piece (Fig. 4).

One of the positive factor influencing the success is sufficient wide surgical exposure. ${ }^{23,24}$ Regardless of the surgical approach used to gain access to the TMJ, the final dissection places the facial nerve at risk for damage. ${ }^{25} \mathrm{~A}$ loss of function of the frontalis and orbicularis oculi muscles is always a possibility. ${ }^{26}$ The incidence of complications such as permanent injury of the facial nerve is very low, ${ }^{7}$ with rate varying from 9 to $18 \%{ }^{27}$ and 1.5 to $32 \%,{ }^{24}$ usually disappearing within 6 months. In this study, modified Myrhaug's, preauricular approach was employed in all 10 cases. Although this incision was used by Myrhaug exclusively to expose the articular eminence for eminectomy

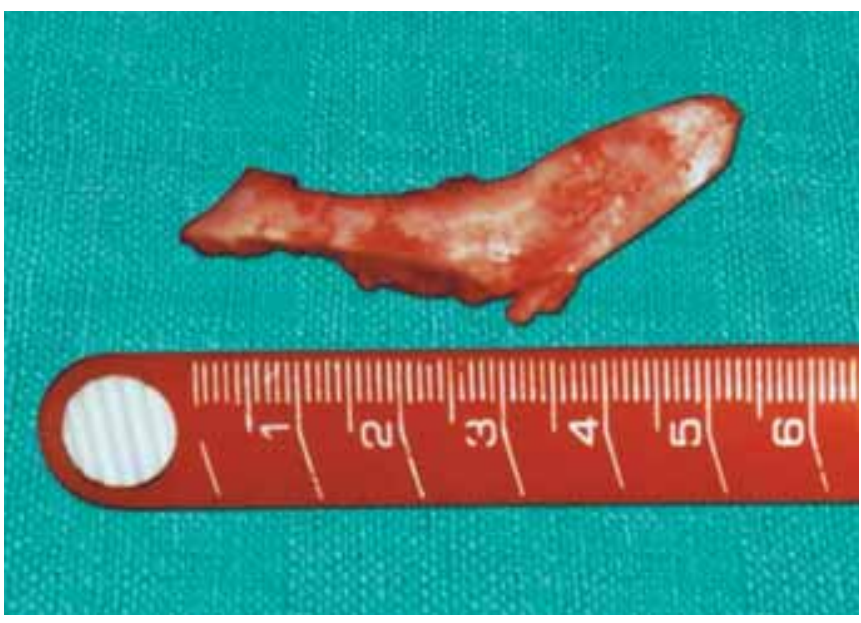

Fig. 4: Osteotomized mass including coronoid process

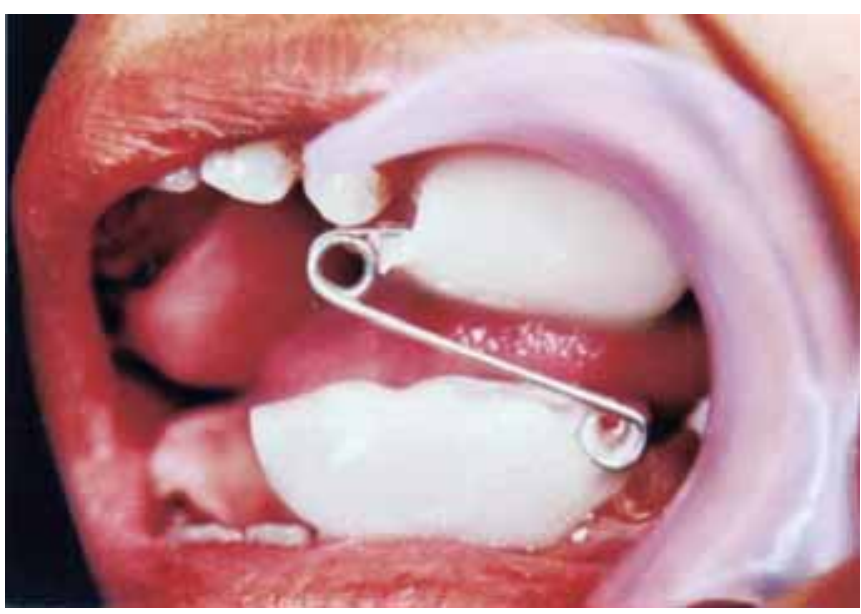

Fig. 5: Custom made intraoral mouth opening exercise device

procedure, it was further modified by extending the anterior part of its horizontal extension slightly forwards and downwards. This incision was advantageous as it allowed excellent visibility of the ankylosed joint along with the exposure of invariably elongated coronoid process and preservation of most of the vital anatomical structures surrounding the joint especially the zygomatic and temporal branches of the facial nerve and massetric nerves and vessels.

After surgical resection, aggressive physiotherapy as recommended was done. Many researchers have also stressed the importance of early mobilization and aggressive physiotherapy for successful treatment. 2,6,12,13,22,28

The existing conventional jaw exerciser has 2 long beaks attached anteriorly to both the jaws as shown and is a cumbersome appliance and hence has low acceptability to the patient leading to reunion and ankylosis of the joint. For aggressive physiotherapy, we used custom made intraoral mouth opening exercise device (Fig. 5). This appliance is constructed to deliver more forces in the posterior segment of the jaw. It is more esthetically designed 
resulting in better acceptability, appreciation and improved compliance of the patient.

In this study, all the patients were encouraged to move the jaw postoperatively as early as possible by taking solid foods and chewing gum to give exercise to the masticatory muscles. Regular exercises were instituted starting from 48 hours postoperatively and were continued for a period of 6 to 8 months.

\section{CONCLUSION}

Creation of sufficient gap at the ankylosed site by removing adequate bone by modified gap arthroplasty gave successful results in the follow-up period and eliminated the need of separate coronoidectomy.

The use of modified Myrhaug's preauricular approach, provided excellent visibility particularly of the elongated coronoid process, minimized bleeding, prevented damage to the vital anatomical structures surrounding the joint and gave acceptable cosmetic results.

However, the ultimate success was also dependant to a large extent on proper mouth opening exercise instituted in the immediate postoperative period continued for at least 6 to 8 months.

\section{REFERENCES}

1. Long X, Li X, Cheng Y, Yang X, Qin L, Qiao Y, et al. Preservation of disc for treatment of traumatic temporomandibular joint ankylosis. J Oral Maxillofac Surg Jul 2005;63(7):897-902.

2. Kaban LB, Perrott DH, Fisher K. A protocol for management of temporomandibular joint ankylosis. J Oral Maxillofac Surg 1990;48:1145-51.

3. Piero C, Alessandro A, Giorgio S, Paolo A, Giorgio I. Combined surgical therapy of temporomandibular joint ankylosis and secondary deformity using intraoral distraction. J Craniofac Surg 2002;13:401-09.

4. Mangenollo-Souza LC, Mariani PB. Temporomandibular joint ankylosis: Report of 14 cases. Int J Oral Maxillofac Surg 2003; 32:24-29.

5. Belmiro Cavalcanti do Egito Vascuniclos, Gabriela Granja Porto, et al. Treatment of temporomandibular joint ankylosis by gap arthroplasty. Med Oral patol Oral Cir Bucal 2006;11:E 66-69.

6. Su-Gwan K. Treatment of temporomandibular joint ankylosis with temporalis muscle and fascia flap. Int J Oral Maxillofac Surg Jun 2001;30(3):189-93.

7. Güven O. Treatment of temporomandibular joint ankylosis by a modified fossa prosthesis. J Craniomaxillofac Surg Aug 2004;32(4):236-42.

8. Lei Z. Auricular cartilage graft interposition after temporomandibular joint ankylosis surgery in children. J Oral Maxillofac Surg Sep 2002;60(9):985-87.

9. Valentini. Interpositional material to prevent reankylosis. Journal of Craniofacial Surgery Jan 2002;13(1):59-67.

10. Roychoudhury A, Parkash H, Trikha A. Functional restoration by gap arthroplasty in temporomandibular joint ankylosis:
A report of 50 cases. Oral Surg Oral Med Oral Pathol Oral Radiol Endod Feb 1999;87(2):166-69.

11. Erdem E, Alkan A. The use of acrylic marbles for interposition arthroplasty in the treatment of temporomandibular joint ankylosis: Follow-up of 47 cases. Int J Oral Maxillofac Surg Feb 2001;30(1):32-36.

12. Sawhney CP. Bony ankylosis of the temporomandibular joint: Follow-up of 70 patients treated with arthroplasty and acrylic Spacer interposition. Plast Reconstr Surg 1986;77:29-38.

13. Das UM, Keerthi R, Ashwin DP, Subramanian R Venkata, Reddy D, Shigggaon N. Ankylosis of temporomandibular joint in children. Journal of Indian Society of Pedodontics and Preventive Dentistry, Case Report 2009;27(2):116-20.

14. Vasconcelos BC, Bessa-Nogueira RV, Cypriano RV. Treatment of temporomandibular joint ankylosis by gap arthroplasty. Med Oral Patol Oral Cir Bucal Jan 2006;11(1):E66-69.

15. Ramezanian M, Yavary T. Comparison of gap arthroplasty and interpositional Gap arthroplasty on the temporomandibular joint ankylosis. Acta Medica Iranica 2006;44(6):391-94.

16. Matsuura H, Miyamoto H, Ogi N, Kurita K, Goss AN. The effect of gap arthroplasty on temporomandibular joint ankylosis: An experimental study. Int J Oral Maxillofac Surg Oct 2001;30(5):431-37.

17. Saeed NR, Kent JN. A retrospective study of the costochondral graft in TMJ reconstruction. Int J Oral Maxillofac Surg Dec 2003;32(6):606-09.

18. MacIntosh RB. The use of autogenous tissues for temporomandibular joint reconstruction. J Oral Maxillofac Surg Jan 2000;58(1):63-69.

19. Speculand B, Hensher R, Powell D. Total prosthetic replacement of the TMJ: Experience with two systems 1988-1997. Br J Oral Maxillofac Surg Aug 2000;38(4):360-69.

20. Shadab M, Pant MC. Role of computed tomography in TM joint problems. Ind J of Oral and Maxillofacial Surg 1991;6:3.

21. Hakim IE, Metwalli SA. Imaging of temporomandibular joint ankylosis. A new radiographic classication. Dentomaxillofacial Radiology 2002;31:19-23.

22. Guven O. Treatment of temporomandibular joint ankylosis by a modified fossa prosthesis. J Craniomaxillofac Surg 2004; 32:236-42.

23. Yoon HJ, Kim HG. Intraoral mandibular distraction osteogenesis in facial asymmetry patients with unilateral temporomandibular joint bony ankylosis. Int J Oral Maxillofac Surg 2002;31: 544-48.

24. Mercuri LG, Auspach WE: Principles for the revision of total Alloplastic TMJ prostheses. Int J Oral Maxillofac Surg 2003;32: 353-59.

25. Politi M, Toro C, Cian R, Costa F, Robiony M. The deep subfascial approach to the temporomandibular joint. J Oral Maxillofac Surg Sep 2004;62(9):1097-1102.

26. Schobel G, Millesi W, Watzke IM, Hollmann K. Ankylosis of the temporomandibular joint. Follow-up of thirteen patients. Oral Surg Oral Med Oral Pathol Jul 1992;74(1):7-14.

27. Weinberg S, Kryshtalskyj B. Facial nerve function following temporomandibular joint surgery using the preauricular approach. J Oral Maxillofac Surg Oct 1992;50(10):1048-51.

28. Qudah MA, Qudeimat MA, Al-Maaita J. Treatment of TMJ ankylosis in Jordanian children: A comparison of two surgical techniques. J Craniomaxillofac Surg 2005;33:30-36. 


\section{ABOUT THE AUTHORS}

\section{Arvind Agarwal (Corresponding Author)}

Professor and Head, Department of Oral and Maxillofacial Surgery Narsinhbhai Patel Dental College and Hospital, Visnagar, Gujarat India, Phone: 9327013779, e-mail:ahmedabaddental@yahoo.com

\section{Brijesh Ruparelia}

Professor and Head, Department of Prosthodontics, Narsinhbhai Patel Dental College and Hospital, Visnagar, Gujarat, India

\section{Ajay Kubawat}

Professor and Head, Department of Orthodontia, Narsinhbhai Patel Dental College and Hospital, Visnagar, Gujarat, India

\section{Sandeep Patel}

Professor and Head, Department of Periodontia, Narsinhbhai Patel Dental College and Hospital, Visnagar, Gujarat, India

\section{Shalini Gupta}

Professor, Department of Periodontia, Faculty of Dental Science Dharamsinh Desai University, Nadiad, Gujarat, India 